\title{
Recent approaches in the development of encapsulated delivery systems for probiotics.
}

\begin{abstract}
The concept of probiotics has been well-known for more than a century. The availability and survival of the consumed probiotics in the colon has not been proved convincingly and needs further studies and clarification. It was not known whether the fastidious probiotics could reach the targeted site of action due to gastrointestinal stress. However, probiotics must sustain themselves in high number, survive during passage through the stomach to the intestine, and react symbiotically with the host when they reach the colon. This review consolidates some of the recent findings and new strategies on the development of a delivery system for targeted colonic delivery of probiotics.
\end{abstract}

Keyword: Probiotic; Delivery system; Microencapsulation; Gastrointestinal transit. 